\title{
Boolean hypercubes and the structure of vector spaces
}

\author{
Ramon Carbó-Dorca ${ }^{a^{*}}$ \\ ${ }^{a}$ Institut de Química Computacional i Catàlisi / Universitat de Girona/ Girona 17071 (Catalonia) Spain \\ *Corresponding author E-mail: ramoncarbodorca@gmail.com
}

\begin{abstract}
Article Info
Keywords: Vector spaces, vector semispaces, vector polyhedra, boolean hypercubes, vector space wireframe, perfect, whole and hollow vectors, complete sum of a vector, inward vector product, inward vector power, power basis sets, generalized scalar products, generalized vector norms, statisticallike vectors and indices

2010 AMS: 15A03, 15A63, 15A72.

Received: 4 April 2018

Accepted: 16 May 2018

Available online: 27 May 2018
\end{abstract}

\begin{abstract}
The present study pretends to describe an alternative way to look at Vector Spaces as a scaffold to produce a meaningful new theoretical structure to be used in both classical and quantum QSPR. To reach this goal it starts from the fact that N-Dimensional Boolean Hypercubes contain as vertices the whole information maximally expressible by means of strings of $\mathrm{N}$ bits. One can use this essential property to construct the structure of $N$ Dimensional Vector Spaces, considering vector classes within a kind of Space Wireframe related to a Boolean Hypercube. This way of deconstruct-reconstruct Vector Spaces starts with some newly coined nomenclature, because, through the present paper, any vector set is named as a Vector Polyhedron, or a polyhedron for short if the context allows it. Also, definition of an Inward Vector Product allows to easily build up polyhedral vector structures, made of inward powers of a unique vector, which in turn one might use as Vector Space basis sets. Moreover, one can construct statistical-like vectors of a given Vector Polyhedron as an extended polyhedral sequence of vector inward powers. Furthermore, the Complete Sum of a vector is defined simply as the sum of all its elements. Once defined, one can use it to compute, by means of inward products, generalized scalar products, generalized vector norms and statistical-like indices attached to a Vector Polyhedron.
\end{abstract}

\section{Introduction}

It will be useless to provide an exhaustive list of literature devoted to vector spaces and their applications. Therefore, just a simplified list containing references which have been useful to the present author will be given. Obviously enough the following quotations, see for instance [1]-[8], contain reference descriptions of vector spaces for learning purposes and practical application within linear algebra algorithms as well. In chemistry one of the main uses vector spaces are employed corresponds to the description of molecules described as vectors, both in discrete and infinite dimensional spaces. In fact, the fields of classical and quantum QSPR studied within the so-called molecular spaces are effectively connected with finite dimensional vector spaces, see as a recent example, references [9]-[28].

Considering the previous reflections, it seems interesting for QSPR purposes and beyond, to study vector spaces from a new point of view, which as far as the author knows has not been described in the current literature. Therefore, one might simply describe the aim of the present study as an essay to deconstruct-reconstruct vector spaces, within the framework of Boolean Hypercubes of the same dimension.

The provided references correspond to many topics, which the author has developed mainly in the 21 st century if they were reflecting in some way or another the picture of vector spaces as are discussed here. See for example references [10]-[22].

Along this paper will be used the well-known Dirac notation to represent vectors. According to this choice, $N$-Dimensional column vectors will be written as: $|\mathbf{a}\rangle=\left(a_{1}, a_{2}, \ldots, a_{N}\right)^{T}=\left\{a_{I} \mid I=1, N\right\}$ and the associated dual row vectors as: $\langle\mathbf{a}|=\left(a_{1}, a_{2}, \ldots, a_{N}\right)$.

An $N$-Dimensional vector space defined over some scalar numerical set $\mathrm{S}$ will be represented by: $V_{N}(S)$.

Symbolized by $\mathbf{H}_{N}$ an N-Dimensional Boolean Hypercube is described by a set of $2^{N}$ vertices, which might be also noted by: $\left\{\left|\mathbf{h}_{I}\right\rangle \mid I=0,2^{N}-1\right\}$. Each vertex is made by all the possible combinations of sets of $N$ binary digits. That is, sets in the form of vectors of $\mathrm{N}$ elements just made by two number-symbols as: $B=\{0,1\} . \mathbf{H}_{N}$ might be seen as the unique set of distinct vector elements, which can be contained within a vector space defined as: $V_{N}(B)$. 


\subsection{Vector spaces and vector semispaces}

By a vector semispace ${ }^{1}$ it is here understood a vector space where within it are not included vectors with negative elements at all, see references [10]-[22]. for an exposition of definitions, properties and applications of semispaces. One must only note the addition in a semispace forms a semigroup, and from this property, the name semispace appears coherently defined. Scalars intervening in the product of a vector by a scalar in semispaces are also considered positive definite.

In principle, it will be latter studied the fact that, to construct any vector space, one only needs vectors strictly bearing non-null elements. Such kind of vectors will be named here: whole vectors.

The set of the positive definite whole vectors in a vector space constitutes a vector semispace.

Broadly speaking, one can consider vector semispaces, as defined over the positive real, rational or natural numbers. One can consider natural vector spaces $V_{N}(\mathbb{N})$, defined over the set of natural numbers, as recently discussed, see references [23]-[24]., directly constructed in the guise of semispaces.

\subsection{Vector polyhedra}

In the present work, by a Vector Polyhedron (or shortly: Polyhedron) $P$ it will be understood a set of M vectors:

$$
P=\left\{\left|\mathbf{p}_{I}\right\rangle=\left(\begin{array}{llll}
p_{1 I} & \left.\left.p_{2 I} \quad \cdots \quad p_{N I}\right)^{T} \mid I=1, M\right\}
\end{array}\right.\right.
$$

belonging to a vector space, that is: $P \subset V_{N}(S)$. The vector elements of a Polyhedron might be named as vertices.

Whenever the value $M>N$, the Polyhedron will be made of linearly dependent vertices. Only when $M \leq N$ there is a possibility that the Polyhedron might be strictly made by a set of linearly independent vertices.

The Gram matrix $\mathbf{G}$ of a Polyhedron $P$ contains the scalar products of the Polyhedron vertices pairs. That is, $\mathrm{G}$ is a symmetrical $(M \times M)$ matrix defined as:

$$
\mathbf{G}=\left\{G_{I J}=\left\langle\mathbf{p}_{I} \mid \mathbf{p}_{J}\right\rangle=\sum_{K=1}^{N} p_{K I} p_{K J} \mid I, J=1, M\right\}
$$

The notation $\mathbf{G}[P]$ makes explicit, if necessary, the fact that the Gram matrix is computed over the vertices of the Polyhedron $P$. Any vector Polyhedron can be considered as constructed by a linearly independent set of vertices whenever: $\operatorname{Det}|\mathbf{G}| \neq 0$.

Vector polyhedra concept has been recently employed in various publications dealing with several topics, see for instance references [22]-[26].

\section{Boolean hypercubes as vector space wireframes}

\subsection{Definition and characteristics of a boolean hypercube}

In the present discussion, each vertex contained in a Boolean Hypercube $\mathbf{H}_{N}$ will be considered as made by all the possible combinations of the digits $B=\{0,1\}$ taken in batches of $N$ terms, which in turn can be depicted as vectors, considered as Boolean Hypercube vertices.

One can alternatively accept the two digits forming the set $\mathrm{B}$ as binary, integer, rational or real numbers, depending on the mathematical context where the Boolean Hypercube is used. For more information about the use which has been made of Hypercubes to study different theoretical and practical problems, see references [27]-[32].

For instance, taken $B$ as the elementary binary digit set or bits, then the $\mathbf{H}_{N}$ vertices become a collection of binary strings, which one can translate into a natural numerical form in the customary manner.

Within this strict Boolean interpretation, each one of the $2^{N}$ vertices of $\mathbf{H}_{N}$ represents a decimal natural number, which, if necessary, one can represent by the transformations:

$$
\forall I=0,2^{N}-1 \Rightarrow I=v\left[\left|\mathbf{h}_{I}\right\rangle\right] \leftrightarrow\left|\mathbf{h}_{I}\right\rangle=\beta[I]
$$

As previously stated any $\mathbf{H}_{N}$ constructed in this fashion is equivalent to a vector space of the same dimension: $V_{N}(B)$, associated to the basic binary number set: $B$.

\subsection{Characteristic vertices of a boolean hypercube}

Two vertices are present in any Boolean Polyhedron: $\mathbf{H}_{N}$, representing two extreme bit constructs. Namely,

a) The zero vertex: $|\mathbf{0}\rangle=(0,0, \ldots, 0)^{T}$ containing an $\mathrm{N}$ times repeated string of the digit $\{0\}$, whose decimal representation one might accept as the number zero, that is: $0=v[|\mathbf{0}\rangle]$.

b) The Mersenne or unity vertex: $|\mathbf{1}\rangle=(1,1, \ldots, 1)^{T}$, possess their components made by the digit $\{1\}$ repeated $\mathrm{N}$ times, containing the decimal representation of the Mersenne number $\mu(N)$, defined as: $\mu(N)=2^{N}-1=v[|\mathbf{1}\rangle]$. The Mersenne number is the largest decimal representation, which can be associated to any bit string contained within the $N$-Dimensional Boolean Hypercube: $\mathbf{H}_{N}$.

Mersenne numbers have relative importance as some are prime numbers, like 7 . The largest prime number computed up to date ${ }^{2}$, is also a Mersenne number: $2^{77,232,917}-1$

\footnotetext{
${ }^{1}$ In some literature, vector semispaces are also known as orthants.

${ }^{2}$ See, for example: https://www.mersenne.org/primes/press/M77232917.html
} 


\subsection{Canonical vertex set}

A set of vertices contained into every $\mathbf{H}_{N}$ is also worth of mention: the canonical vertex set, E, which one might define using the representation:

$$
E=\left\{\left|\mathbf{e}_{I}\right\rangle \mid I=0, N-1\right\} \equiv\left\{\left|\mathbf{h}_{J}\right\rangle\left|J=2^{I}\right| I=0, N-1\right\}
$$

where each element of E could be associated to the decimal powers of two using the transformation:

$$
\forall I=0, N-1: v\left[\left|\mathbf{e}_{I}\right\rangle\right]=2^{I}
$$

From the Boolean point of view, each element of the canonical vertex set $E$ corresponds to a string of $N-1$ digits $\{0\}$ and just one digit $\{1\}$, which one can consider positioned into every one of the elements of the associated $N$-Dimensional vector. The vector set $E$, viewed as an integer, rational or real construct, corresponds to the so-called canonical basis set, which one can suppose as belonging to any vector space of the appropriate dimension. Also, any vertex of $\mathbf{H}_{N}$ corresponds to a linear combination of the canonical vertex set, whose coordinates are coincident with the elements of the considered vertex in question.

In terms of the nomenclature used in many previous papers and adopted here, one might consider the canonical basis set as a vector Polyhedron. Because such a general name, as commented in the introduction, has been chosen to be applied to any kind of vector set, belonging to any vector space.

Such a nomenclature, as used here, seems preferable to the alternative possible name of vector Polytope. As one might accept, a Polytope being a specific construction case of a regular Polyhedron. Accordingly, one might consider a Hypercube $\mathbf{H}_{N}$ as an element among vector Polyhedra but being regular as an $N$-Dimensional Polytope too.

Finally, one can add a comment about the fact that, while the Mersenne vertex $|\mathbf{1}\rangle \equiv \beta\left[2^{N}-1\right]$ might be considered as the last vertex of some $N$-Dimensional Boolean Hypercube $\mathbf{H}_{N}$, the canonical vertex $\left|\mathbf{e}_{N}\right\rangle \equiv \beta\left[2^{N}\right]$, which corresponds to the next natural number representation in the sequence of binary numbers, might be taken as the first vector of $\mathbf{H}_{N+1}$, which is not contained in $\mathbf{H}_{N}$.

Concatenation $\cup$ is an operation involving Hypercubes, which can be defined to construct Hypercubes of higher dimension from lesser dimensional ones, see for example [30, 31]. That is, for instance, while it can be written: $\{0\} \cup \mathbf{H}_{N} \subset \mathbf{H}_{N+1}$, also one can consider that: $\left|\mathbf{e}_{N}\right\rangle \in \mathbf{H}_{N+1} \wedge\left|\mathbf{e}_{N}\right\rangle \notin\{0\} \cup \mathbf{H}_{N}$.

\subsection{Vertex classes}

One can classify the set of $\mathbf{H}_{N}$ vertices into $N+1$ classes, according to the number of digits $\{0\}$ or $\{1\}$, used to construct the corresponding class. Usually, there are two complementary sets of vertex classes.

Using the digit $\{1\}$ as classifier, one can start with the class holding zero digits $\{1\}$, formed by the zero vector: $|\mathbf{0}\rangle$ only, and end with the class holding $N$ digits $\{1\}$, containing the Mersenne vertex $|\mathbf{1}\rangle$ only. The class of $N$ vertices containing one digit $\{1\}$ and $N-1$ digits $\{0\}$ is coincident with the canonical vertex set $\mathrm{E}$.

One might accept that every $\mathbf{H}_{N}$ class is forming a vector Polyhedron by itself. The classification based on the number of digits $\{1\}$ in every $\mathbf{H}_{N}$ vertex within the class, might be described with the symbols: $\mathbf{C}_{\left[N_{1} ; N_{0}\right]}$, with $\left[N_{1} ; N_{0}\right]$ meaning the number of digits $\{1\}$ and $\{0\}$ respectively, contained in every vector belonging to the class.

Obviously enough: $N_{1}+N_{0}=N$. The number of vertices within each class corresponds to the combinatorial numbers: $\left(\begin{array}{c}N \\ N_{1}\end{array}\right)=\frac{N !}{N_{1} !\left(N-N_{1}\right) !}$, which correspond to the $N$-th row of the Pascal triangle. Following this thought, it must be also obtained: $\sum_{K=0}^{N}\left(\begin{array}{c}N \\ K\end{array}\right)=2^{N}$.

Every vertex class appears to be disjoint with respect of the remnant classes. No vertex class appears closed with respect to the vector sum though.

Complementary classes correspond to classes, which one can describe with the two symbols: $\mathbf{C}_{[K ; L]}$ and $\mathbf{C}_{[L ; K]}$. Complementary vertices belonging to complementary classes are pairs of vertices where the positions of the corresponding binary digits are interchanged: summed up, such kind of vertices produce the Mersenne vertex.

\subsection{The complementary canonical vertex set as a basis set}

The classes $\mathbf{C}_{[K ; N-K]}$ and $\mathbf{C}_{[N-K ; K]}$ are complementary for every value of $K$, with allowed values in the interval: $\{0, N\}$ and $K$ taking here the role of the number of digits $\{1\}$ present within the vertices of the class.

The classes $\mathbf{C}_{[1 ; N-1]} \equiv E$ and $\mathbf{C}_{[N-1 ; 1]} \equiv J$ are thus complementary. In fact, one might gather the canonical vertex set $E$ as the columns of the unit matrix of the appropriate dimension, using a logical Kronecker delta ${ }^{3}$ :

$$
I_{N}=\left\{I_{N ; I J}=\delta(I=J) \mid I, J=0, N-1\right\}=\left(\left|\mathbf{e}_{0}\right\rangle ;\left|\mathbf{e}_{1}\right\rangle ; \ldots\left|\mathbf{e}_{N-1}\right\rangle\right) .
$$

The complementary class $\mathrm{J}$ of the canonical vertex set $\mathrm{E}$ might be written by defining first the unity matrix: $\mathbf{1}_{N}$, as a matrix with all the columns or rows made by the Mersenne vertex repeated $\mathrm{N}$ times:

$$
\mathbf{1}_{N}=\left\{1_{N ; I J}=1 \mid I, J=0, N-1\right\}=(|\mathbf{1}\rangle ;|\mathbf{1}\rangle ; \ldots|\mathbf{1}\rangle)
$$

and then, the complementary matrix to the unit one, can be easily written, using again a logical Kronecker delta, as:

$$
\mathbf{J}_{N}=\mathbf{1}_{N}-\mathbf{I}_{N}=\left\{J_{N ; I J}=\delta(I \neq J) \mid I, J=0, N-1\right\}=\left(\left|\mathbf{j}_{0}\right\rangle ;\left|\mathbf{j}_{1}\right\rangle ; \ldots\left|\mathbf{j}_{N-1}\right\rangle\right) .
$$

\footnotetext{
${ }^{3} \mathrm{~A}$ logical Kronecker delta is defined as a symbol written this way: $\delta(L)$, where L might be any logical expression. Then, this symbol can be used as: $\delta(L \equiv . T$. $)=1$ or $\delta(L \equiv . F)=$.0 . Thus, the classical Kronecker delta might be written as: $\delta(I=J)$.
} 
Finally, one can easily describe the $\mathbf{C}_{[N-1 ; 1]}$ class vertex composition as:

$$
\mathbf{C}_{[N-1 ; 1]} \equiv J=\left\{\left|\mathbf{j}_{I}\right\rangle \mid I=0, N-1\right\}
$$

Therefore, one can name the Polyhedron $J$ as the complementary canonical basis set. This might be so because their elements are linearly independent. One can easily show the linear independence of the set of columns or rows of the vertex class $J$, considering that one might write the unity matrix spectrum as:

$$
\operatorname{Sp}\left(\mathbf{1}_{N}\right)=\left\{\lambda_{1}=N ; \lambda_{K}=0 \mid K=2, N\right\},
$$

then the spectrum of the matrix $\mathbf{J}_{N}$, is easily computed as:

$$
\operatorname{Sp}\left(\mathbf{J}_{N}\right)=\left\{\mu_{1}=N-1 ; \mu_{K}=-1 \mid K=2, N\right\},
$$

therefore:

$$
\operatorname{Det}\left|\mathbf{J}_{N}\right|=(-1)^{N-1}(N-1)
$$

Showing the fact that the vertices in the class $J$ are linearly independent, therefore can be considered as a basis set complementary to the canonical basis set.

\subsection{Model or seed vertex of a class $\mathbf{C}_{\left[N_{1} ; N_{0}\right]}$ in $\mathbf{H}_{N}$}

Every class holding $N_{1}$ digits $\{1\}$ and thus the corresponding $N_{0}$ digits $\{0\}$ possess a vertex, which might be called as the model (or seed) vertex: $\left|\mathbf{c}_{\left[N_{1} ; N_{0}\right]}\right\rangle$, which can be defined as a vector with $N_{1}$ ones in the first places counted from the top and the rest filled by $N_{0}$ zeroes. One can express this ordering of the binary digits leading to the model vertex of the class $\mathbf{C}_{\left[N_{1} ; N_{0}\right]}$ by:

$$
\left|\mathbf{c}_{\left[N_{1} ; N_{0}\right]}\right\rangle=\left(\begin{array}{c}
\left|\mathbf{1}_{N_{1}}\right\rangle \\
\left|\mathbf{0}_{N_{0}}\right\rangle
\end{array}\right)
$$

\subsubsection{Mersenne basis set}

The set of model vertices in each Boolean Hypercube $\mathbf{H}_{N}$, that is the sequence:

$$
I=1, N:\left|\mathbf{c}_{[I ; N-I]}\right\rangle=\left(\begin{array}{c}
\left|\mathbf{1}_{I}\right\rangle \\
\left|\mathbf{0}_{N-I}\right\rangle
\end{array}\right)
$$

where the elements of the sequence contain in a first place the sequence of Mersenne vertices of dimension I: $I=1, N:\left|\mathbf{1}_{I}\right\rangle$. Conveniently arranged as columns of a square, upper triangular, $(N \times N)$ matrix as follows:

$$
\mathbf{M}_{N}=\left\{M_{I J}=\delta(I \leq J) \mid I, J=1, N\right\},
$$

yield a non-singular matrix. This is so as Det $\left|\mathbf{M}_{N}\right|=1$, resulting from the fact that the diagonal of the matrix $\mathbf{M}_{N}$ coincides with the unit matrix, that is, one can write: $\operatorname{Diag}\left(\mathbf{M}_{N}\right)=\mathbf{I}_{N}$. Thus the matrix $\mathbf{M}_{N}$ will be named here Mersenne matrix for obvious reasons.

Because of the non-singularity of the Mersenne matrix, its columns correspond to a new basis set polyhedron: $M$, contained in the Boolean Hypercube $\mathbf{H}_{N}$ and constructed by the Mersenne vertices of the increasing dimensions from 1 to $N$, which can be associated to the non-zero model vertices, contained into any Boolean Hypercube $\mathbf{H}_{N}$.

Contrarily to the previously discussed basis sets, which were found forming a whole Boolean Hypercube class, the Mersenne basis set is made of the whole collection of model vertices of the available non-zero classes.

\subsubsection{The structure of class elements}

The rest of the class vertex members can be obtained via the set of corresponding combinations of the digits $\{1\}$ and $\{0\}$ present in the model vertex. Every combination of the model vertex elements produces a vertex contained into the well-defined class $\mathbf{C}_{\left[N_{1} ; N_{0}\right]}$. One might refer to the elements of a given class as the collection of shadow vertices or shadow vectors of the model vertex.

However, independently of the class construction, one can see, from considering the corresponding model vertex $\left|\mathbf{c}_{\left[N_{1} ; N_{0}\right]}\right\rangle$, that one can also obtain all the shadow vectors within a given class, as generated by some transformation of the type:

$$
\forall K=1,\left(\begin{array}{c}
N \\
N_{1}
\end{array}\right): T_{K}\left(\left|\mathbf{c}_{\left[N_{1} ; N_{0}\right]}\right\rangle\right)=\left|\mathbf{c}_{\left[N_{1} ; N_{0}\right] ; K}\right\rangle \in \mathbf{C}_{\left[N_{1} ; N_{0}\right]},
$$

producing all the necessary combinations of the digits $\{1\}$ and $\{0\}$, and thus forming the vertices of $\mathbf{H}_{N}$ belonging to the class. Of course, the transformation $T_{1}$ corresponds to the unit operator.

One can further use this previously defined transformation of the model vector: $\left|\mathbf{c}_{\left[N_{1} ; N_{0}\right]}\right\rangle$, as an algorithm providing the ways on how a Mersenne vector $\left|\mathbf{1}_{N_{1}}\right\rangle$, belonging in fact to a lesser dimensional Boolean Hypercube $\mathbf{H}_{N_{1}}$, (that is: $N_{1}<N$ ) could be transformed into the shadow vertices belonging to a given class in $\mathbf{H}_{N}$. This is due that one can write: $\left|\mathbf{c}_{\left[N_{1} ; N_{0}\right]}\right\rangle=\left|\mathbf{1}_{N_{1}}\right\rangle \cup\left|\mathbf{0}_{N_{0}}\right\rangle$. 


\subsection{Whole and hollow vertices and vectors}

Considering the previous definitions and characteristics of the Boolean Hypercube vertices, one can propose the following definitions. In fact, from analyzing at the light of what has been said in the previous paragraphs, from observing all the vertices contained within a given $N$-Dimensional Boolean Hypercube, one can realize the unique vertex possessing the characteristic of being a truly $N$-Dimensional vector is the Mersenne or unity vertex $\left|\mathbf{1}_{N}\right\rangle$.

Shadow vertices in each class $\mathbf{C}_{\left[N_{1} ; N_{0}\right]}$ are obviously related to the model vertex of this class, via the transformation discussed in the previous paragraph. Thus, all of them might be associated to the elements of a Mersenne vertex $\left|\mathbf{1}_{N_{1}}\right\rangle$ of a lesser dimensional Boolean Hypercube $\mathbf{H}_{N_{1}}$. The Mersenne vertices of Boolean Hypercubes might be associated to the kind of vectors in vector spaces, which one has previously named as whole vectors. Remembering that one can use such adjective to giving name to vectors of a Polyhedron collection in a specific $N$-Dimensional vector space, possessing no null elements at all. The whole vectors correspond to the true $N$-Dimensional elements of such a space.

One can call vectors associated to any other class, bearing any number of null elements as hollow vectors.

Therefore, Boolean Hypercube classes can be associated to the vectors which can be termed as hollow, whenever bearing one or more digits $\{0\}$. Hollow vectors, depending of the number of null elements, $N_{0}$, can be associated to the corresponding Boolean Hypercube $\mathbf{H}_{N}$ shadow vector elements attached to the class: $\mathbf{C}_{\left[N_{1} ; N_{0}\right]}$.

\section{Perfect vectors and generation of vector semispace elements}

Realizing the nature of the whole vectors which can be found in a semispace, one can also consider the vectors, which are ordered canonically reversed $^{4}$, from maximal to minimal elements. That is, if one defines a whole vector of a semispacelike:

$$
|\mathbf{a}\rangle=\left\{a_{I} \mid I=1, N\right\} \wedge \forall I: a_{I} \neq 0 .
$$

Then, such a whole vector when chosen with canonically reversed ordered elements, might be also named a perfect vector, considering that:

$$
a_{1}>a_{2}>\ldots>a_{N} .
$$

Therefore, initially one can first suppose a perfect semispace as a subset of a semispace constructed from perfect vectors, that is: canonically reverse ordered positive definite whole vectors.

Then, one might construct the entire semispace via the $N$ ! permutations of each positive definite perfect vector.

That is, suppose such a perfect vector known, then one can define the permutation operators' collection in the form: $\left\{P_{K} \mid K=1, N\right.$ ! $\}$, such that:

$$
\forall K=1, N !:\left|\mathbf{a}_{K}\right\rangle=P_{K}(|\mathbf{a}\rangle),
$$

being every element of the vector permutation Polyhedron:

$$
A=\left\{\left|\mathbf{a}_{K}\right\rangle \mid K=1, N !\right\}
$$

a whole vector deduced from a perfect vector $|\mathbf{a}\rangle$.

That is: the elements of every permutation vector $\left|\mathbf{a}_{K}\right\rangle$ might be considered reordered according to a permutation of the elements with the canonically ordered subindices of the perfect vector acting as a seed.

Hollow vectors do not need to be considered, as they can be formed as elements in lesser dimensional semispaces, and constructed by a lesser dimensional perfect vector, which one might transform into a hollow vector via the definition of a corresponding model vertex with the necessary number of digits $\{0\}$ followed by the described combination procedure.

Moreover, from a perfect vector defined in an $N$-Dimensional vector space, one can also deduce the $N$ vertices of a circular Polyhedron, constructed by choosing the $N$ circular permutations of the original perfect vector acting as a seed.

\subsection{Signature hypercubes and the construction of vector spaces from a unique whole vector}

Consider a Boolean Hypercube: $\mathbf{H}_{N}=\left\{\left|\mathbf{h}_{I}\right\rangle \mid I=0,2^{N}-1\right\}$. One can readily compute the centroid $\left|\mathbf{h}_{C}\right\rangle$ of such a Boolean Hypercube, employing the arithmetic mean of its vertices.

Such vector mean might be considered as yielding a homothetic vector of the Mersenne vertex:

$$
\left|\mathbf{h}_{C}\right\rangle=2^{-N} \sum_{I=0}^{2^{N}-1}\left|\mathbf{h}_{I}\right\rangle=2^{-1}|\mathbf{1}\rangle,
$$

that is: obtaining in this way the Hypercube centroid as corresponding to a homothetic Mersenne vertex scaled by a factor $\frac{1}{2}$.

One can origin shift the Boolean Hypercube $\mathbf{H}_{N}$ using the centroid $\left|\mathbf{h}_{C}\right\rangle$, producing in such a manner a new Polyhedron construct, which might be called Signature Hypercube: $\mathbf{S}_{N}=\left\{\left|\mathbf{S}_{I}\right\rangle \mid I=0,2^{N}-1\right\}$, and one might symbolically express it via the algorithm:

$$
\mathbf{S}_{N}=2\left(\mathbf{H}_{N}-\frac{1}{2}|\mathbf{1}\rangle\right) \Rightarrow \forall I=0,2^{N}-1:\left|\mathbf{s}_{I}\right\rangle=2\left(\left|\mathbf{h}_{I}\right\rangle-\frac{1}{2}|\mathbf{1}\rangle\right) \equiv 2\left|\mathbf{h}_{I}\right\rangle-|\mathbf{1}\rangle .
$$

Therefore, the new Signature Hypercube $\mathbf{S}_{N}$ possess its vertices made by the digits: $\{-1 ;+1\}$. The vertices of $\mathbf{S}_{N}$ correspond to any combination of signs, which might be called a signature, which can be associated in turn to any $N$-dimensional positive definite whole vector. The signature vectors have been already described in another context, see for example reference [33].

\footnotetext{
${ }^{4}$ Perfect vectors, of course, could also be chosen canonically ordered from minimal to maximal elements. But here have been chosen in reverse order to keep some resemblance to the ordered definition of model vertices in Boolean Hypercubes classes.
} 
Then, to every perfect vector in a semispace, as previously commented, one can attach a set of permutations of its elements, yielding $N$ ! whole vectors. Afterwards, from every permuted whole vector constructed in this way, one can build an additional set of $2^{N}$ vectors bearing the corresponding signatures, attaching the signs of every vertex in $\mathbf{S}_{N}$ to the positive definite whole vector elements.

Consequently, to every perfect vector of a semispace, one can easily construct as many as $M(N)=2^{N}(N$ !) different vectors.

For example, considering a $N=10$ dimensional vector semispace, for each perfect vector belonging to it, one can build a set of 3715891200 vectors belonging to the 10-Dimensional vector space. That is, some Polyhedron made of a large amount of vector elements, might be generated from only one perfect vector as a seed.

\subsection{Vector classes}

The class patterns of the Boolean Hypercube vertices might be used to describe both hollow and whole vectors. First, hollow vectors will be analyzed and then whole vectors will be classified. In fact, the relevant result of this paragraph can be resumed by the statement that, in any vector space the structure which matters is just the set of whole vectors.

\subsubsection{Hollow vector classes}

To start with, one can consider the hollow vectors first. Any hollow vector can have from one to all the elements null. This last occurrence will define the zero vector of the space, considered coincident with the zero vertex $|\mathbf{0}\rangle$ of the corresponding Boolean Hypercube.

Therefore, it can be accepted that hollow vectors can be also gathered into classes, which can be noted in turn as: $\left\{\mathbf{L}_{[K]} \mid K=0, N\right\}$. The subindex between brackets meaning the number of zero elements present anywhere in the vector.

Of course, the class $\mathbf{L}_{[0]}$ contains all the whole vectors and has been considered as a hollow class just for the sake of completeness.

Any vector $|\mathbf{a}\rangle$ belonging to a hollow class $\mathbf{L}_{[K]}$ can be referred to a vector seed, belonging in turn to some Boolean Hypercube class of the type: $\mathbf{C}_{[N-K, K]}$. The difference in both type of classes is such that the unity vector $\left|\mathbf{1}_{N-K}\right\rangle$ has been substituted say, by a whole perfect vector of dimension $N-K:\left|\mathbf{a}_{N-K}\right\rangle$.

Within the vector $\left|\mathbf{a}_{N-K}\right\rangle$ all the non-zero vector elements are gathered in the same order as in the related hollow vector of $\mathbf{C}_{[N-K, K]}$. The decomposition of the original vector into the whole vector above and a hollow vector, that is, like:

$$
|\mathbf{a}\rangle \Rightarrow\left(\begin{array}{c}
\left|\mathbf{a}_{N-K}\right\rangle \\
\left|\mathbf{0}_{K}\right\rangle
\end{array}\right)
$$

where the zeroes are gathered at the bottom of the vector, makes it possible to consider the resultant reordered vector as the seed of the hollow class $\mathbf{L}_{[K]}$.

The remaining elements of the class could be deduced from the elements of the Boolean Hypercube class $\mathbf{C}_{[N-K, K]}$, substituting the unit elements of such class by the elements of the condensed $(N-K)$-Dimensional whole vector $\left|\mathbf{a}_{N-K}\right\rangle$.

Therefore, hollow vectors might be considered as whole vector elements of a $(N-K)$-Dimensional subspace. That is: in fact, hollow vectors might be considered as whole vectors contained into a lower dimensional space.

Consequently, the analysis of the elements of any vector space essentially might refer to the study of its whole vectors.

\subsubsection{Whole vectors}

Whole vectors might be classified into a set of classes, which can be referred to the Boolean Hypercube classes, in a similar way as the hollow vectors have previously been studied.

Whole vectors might have elements only repeated once, thus producing upon canonical or reverse canonical ordering a perfect vector, while the next alternative possibilities might consist into an element repeated twice, thrice... and so on. The last possible occurrence is having an element repeated $N$ times, producing an homothecy of the Mersenne vertex, or a whole vector like: $|\mathbf{a}\rangle=\alpha|\mathbf{1}\rangle$.

Therefore, in this manner one can describe a set of whole vectors classes too, which can be described as: $\left\{\mathbf{W}_{[K]} \mid K=1, N\right\}$. Any vector in a class $\mathbf{W}_{[K]}$ can be associated to the Boolean Hypercube class: $\mathbf{C}_{[K, N-K]}$. Constructed with the Mersenne part of the model class vector, substituted by the repeated element $\alpha$ multiplying the same Mersenne vector: $\left|\mathbf{1}_{K}\right\rangle$ and the bottom part formed by a whole vector $\left|\mathbf{a}_{N-K}\right\rangle$ bearing the non-repeated elements disposed in the same order as in the original vector. Or what is the same:

$$
|\mathbf{a}\rangle \Rightarrow\left(\begin{array}{c}
\alpha\left|\mathbf{1}_{K}\right\rangle \\
\left|\mathbf{a}_{N-K}\right\rangle
\end{array}\right)
$$

an arrangement which, can be considered as the model vector of the whole class $\mathbf{W}_{[K]}$.

The original vector might be reordered as any of the other members of the class, just considering the shadow vertex of $\mathbf{C}_{[K, N-K]}$, where the original digits $\{1\}$ might be substituted by the repeated elements and the original Boolean Hypercube digits $\{0\}$, exchanged by the original non-repeated elements of the vector.

As any homothetic vector of the Mersenne vertex corresponds to an element of a monodimensional space, the whole vectors belonging to the class $\mathbf{W}_{[K]}$, might be considered as belonging to a $(N-K+1)$-Dimensional subspace.

Therefore, only whole vectors belonging to the class $\mathbf{W}_{[1]}$ could be considered as true vectors belonging to the vector space $V_{N}(S)$.

\section{Inward vector products and inward vector powers}

Before proceeding with more consequences of the above definitions, a fundamental simple operation: the inward product of two vectors. must be described. 


\subsection{Inward product of two vectors}

In a vector space $V_{N}(S)$ a binary operation noted as a product, the inward product ${ }^{5}$, can be defined between every pair of vectors. Inward products have been described and employed in many places by the author, see for example references [15], [34]-[36].

To visualize such a product of two vectors, one has just to transform the vectors into a pair of diagonal matrices, then execute their product and retransform the resultant diagonal matrix back into a vector.

Suppose two vectors in $V_{N}(S)$ :

$$
\{|\mathbf{a}\rangle,|\mathbf{b}\rangle\} \subset V_{N}(S) \rightarrow|\mathbf{a}\rangle=\left\{a_{I} \mid I=1, N\right\} \wedge|\mathbf{b}\rangle=\left\{b_{I} \mid I=1, N\right\} .
$$

The following algorithm easily defines their inward product:

$$
|\mathbf{a}\rangle *|\mathbf{b}\rangle=|\mathbf{p}\rangle=\left\{p_{I} \mid I=1, N\right\} \in V_{N}(S) \rightarrow \forall I=1, N: p_{I}=a_{I} b_{I}
$$

The inward product is commutative, associative and distributive with respect to the vector summation.

The neutral element for the inward product is made precisely of the Mersenne or unity vector $|\mathbf{1}\rangle$ of the appropriate dimension, because it is trivial to demonstrate that:

$$
\forall|\mathbf{a}\rangle \in V_{N}(S) \rightarrow|\mathbf{a}\rangle *|\mathbf{1}\rangle=|\mathbf{1}\rangle *|\mathbf{a}\rangle=|\mathbf{a}\rangle
$$

As an obvious application example, the generation of the set of signed vectors from a whole vector, as previously discussed, using the vertices of a Signature Hypercube, can be performed via the inward product of the signature vertices by the whole vector of the appropriate dimension. Suppose known the appropriate Signature Hypercube: $\mathbf{S}_{N}=\left\{\left|\mathbf{s}_{I}\right\rangle \mid I=0,2^{N}-1\right\}$ and some whole vector $|\mathbf{a}\rangle \in V_{N}(S)$, the set of all signed vectors, which can be associated to any positive definite whole vector can be simply obtained using:

$$
|\mathbf{a}\rangle \rightarrow I=0,2^{N}-1:\left|\mathbf{a}_{I}\right\rangle=\left|\mathbf{s}_{I}\right\rangle *|\mathbf{a}\rangle .
$$

Obviously enough, the inward vector product can be applied to more than a pair of vectors. Suppose a Polyhedron of some vector space: $P=\left\{\left|\mathbf{a}_{I}\right\rangle \mid I=1, M\right\} \subset V_{N}$, then it can be written a vector resultant of the inward product of the whole Polyhedron vertices:

$$
|\mathbf{p}\rangle=\underset{K=1}{\stackrel{M}{*}}\left|\mathbf{a}_{K}\right\rangle=\left|\mathbf{a}_{1}\right\rangle *\left|\mathbf{a}_{2}\right\rangle * \ldots *\left|\mathbf{a}_{M}\right\rangle \in V_{N} \rightarrow \forall I=1, N: p_{I}=\prod_{K=1}^{M} a_{I K}
$$

\subsection{Inward inverse vectors}

Only whole vectors can be supposedly attached to some true inward inverse vector. That is, whenever $|\mathbf{a}\rangle \in V_{N}(S)$ is a whole vector, then the inward inverse of this vector can be easily defined as the whole vector:

$$
|\mathbf{a}\rangle^{[-1]} \in V_{N}(S) \rightarrow|\mathbf{a}\rangle^{[-1]}=\left\{a_{I}^{-1} \mid I=1, N\right\}
$$

therefore, it is easy to write:

$$
|\mathbf{a}\rangle *|\mathbf{a}\rangle^{[-1]}=|\mathbf{a}\rangle^{[-1]} *|\mathbf{a}\rangle=|\mathbf{1}\rangle
$$

Hollow vectors can be transformed into the model or seed vectors, while their shadow Boolean vector is kept as a class structure token. Once the separated non-zero part of the seed vector is made, then the inward inverse of this whole vector can be computed. This can be followed by a pseudoinverse vector, which can be constructed using the corresponding shadow vector and locating the inverse non-zero elements accordingly into the final vector form.

\subsection{Inward power of a vector}

One of the interesting applications of inward products corresponds to the possibility to compute successive inward powers of a given vector. Suppose known: $\forall|\mathbf{a}\rangle \in V_{N}(S)$, its inward $p$-th power: $|\mathbf{a}\rangle^{\lceil p]}$ can be constructed via the following algorithm:

$$
\left|\mathbf{a}^{[p]}\right\rangle=\underset{k=1}{\stackrel{*}{*}}|\mathbf{a}\rangle=|\mathbf{a}\rangle *|\mathbf{a}\rangle \ldots *|\mathbf{a}\rangle=\left\{a_{I}^{p} \mid I=1, N\right\}
$$

An inward power Polyhedron can be associated to the collection of a given vector and their inward powers up to some fixed limit. For instance:

$$
P_{P}(|\mathbf{a}\rangle)=\left\{\left|\mathbf{a}^{[p]}\right\rangle \mid p=1, P\right\}=\left\{|\mathbf{a}\rangle,|\mathbf{a}\rangle^{[2]}, \ldots|\mathbf{a}\rangle^{[P]}\right\} .
$$

\footnotetext{
${ }^{5}$ Such product is also named as Hadamard or Schur or Diagonal product.
} 


\subsection{Inward function of a vector}

Of course, the inward power allows the construction of inward smooth vector functions, via Taylor series around some point $\left|\mathbf{x}_{0}\right\rangle$, for instance:

$$
|\varphi[\mathbf{x}]\rangle=\left\{\varphi\left(x_{I}\right) \mid I=1, N\right\} \Rightarrow|\varphi[\mathbf{x}]\rangle=\sum_{k=0}^{\infty} \frac{1}{k !}\left(|\mathbf{x}\rangle-\left|\mathbf{x}_{0}\right\rangle\right)^{[k]}\left|\frac{\partial^{k} \varphi\left[\mathbf{x}_{0}\right]}{\partial \mathbf{x}^{[k]}}\right\rangle,
$$

with the auxiliary definition

$$
\left|\frac{\partial^{k} \varphi\left[\mathbf{x}_{0}\right]}{\partial \mathbf{x}^{[k]}}\right\rangle=\left\{\left.\frac{d^{k} \varphi\left(x_{I}\right)}{d x_{I}^{k}}\right|_{x_{I}=x_{I, 0}} \mid I=1, N\right\} .
$$

The notation used here into the vector associated to an inward function of a vector: $|\varphi[\mathbf{x}]\rangle$ has been used to differentiate it from a multivariate scalar function of a vector, which can be written as: $\varphi(|\mathbf{x}\rangle)$.

\section{The making of a generalized Minkowski vector space}

\subsection{Complexity hypercubes}

One might start remembering again the Signature Hypercube: $\mathbf{S}_{N}$, which has been deduced from a Boolean Hypercube $\mathbf{H}_{N}$ of the appropriate dimension. As the vertices of $\mathbf{S}_{N}$ possess elements defined into the two-digit set: $\{-1 ;+1\}$, then one can easily imagine another Hypercube of the same dimension derived from: $\mathbf{S}_{N}$. This can be done just describing the operation which one might call inward square root of a vector:

$$
\forall|\mathbf{a}\rangle=\left\{a_{I} \mid I=1, N\right\} \Rightarrow\left|\mathbf{a}^{\left[\frac{1}{2}\right]}\right\rangle=\left\{\left(a_{I}\right)^{\frac{1}{2}} \equiv \sqrt{a_{I}} \mid I=1, N\right\},
$$

and apply it to each Signature Hypercube vertex, that is:

$$
\mathbf{M}_{N}=\mathbf{S}_{N}^{\left[\frac{1}{2}\right]} \Rightarrow \forall I=0,2^{N}-1:\left|\mathbf{m}_{I}\right\rangle=\left|\mathbf{s}_{I}^{\left[\frac{1}{2}\right]}\right\rangle \equiv\left(2\left|\mathbf{h}_{I}\right\rangle-|\mathbf{1}\rangle\right)^{\left[\frac{1}{2}\right]} .
$$

It is easily seen that the elements of the resultant Complexity Hypercube will be now described by the two digits: $\{\sqrt{-1} ;+1\} \rightarrow\{+i ;+1\}$, where $i=\sqrt{-1}$ corresponds to the imaginary unit.

The Complexity Hypercube acts as another $2^{N}$ element source of an alternative kind of powerful binary attribute, which like signs, can be associated to whole $N$-Dimensional real vectors.

Again, two vectors can be chosen among all the vertices of a complexity Hypercube, the usual Mersenne vertex $|\mathbf{1}\rangle$ and the imaginary Mersenne vertex: $|\mathbf{i}\rangle=i|\mathbf{1}\rangle$, which appears as a pure imaginary homothecy of the Mersenne vertex.

In fact, one can generate from the elements of $\mathbf{M}_{N}$ the Signature Hypercube vertices, using the definition of the inward $p$-th power of a vector and simply considering that the following relationship holds:

$$
\forall I=0,2^{N}-1:\left|\mathbf{m}_{I}^{[2]}\right\rangle=\left|\mathbf{s}_{I}\right\rangle,
$$

just bearing in mind the trivial equality: $i^{2}=-1$. Then, in this sense, the number of vectors which can be deduced from a perfect vector might be supposedly correspond to: $N ! 2^{2 N}$

\subsection{Complex vectors via complexity hypercubes}

Given a perfect $N$-Dimensional real vector $|\mathbf{a}\rangle$, one can therefore construct a complex vector. Thus, using the following algorithm, accordingly to the previous inward product definition:

$$
\forall I=0,2^{N}-1:\left|\mathbf{c}_{I}\right\rangle=\left|\mathbf{m}_{I}\right\rangle *|\mathbf{a}\rangle,
$$

it is obtained the vector set which might be easily considered as a generalized complex number set.

This is so, because it might be stated that a typical complex number, written as: $c=a+i b$, can be expressed as the sum of two one-Dimensional elements, forming the real and imaginary part.

Thinking of a two-dimensional case here, according to what has been previously said about Complexity Hypercubes, one can write four possible complexity attribute vertices as follows:

$$
\left\{\left(\begin{array}{l}
1 \\
1
\end{array}\right),\left(\begin{array}{l}
i \\
1
\end{array}\right),\left(\begin{array}{l}
1 \\
i
\end{array}\right),\left(\begin{array}{l}
i \\
i
\end{array}\right)\right\}
$$

Every one of these vertices produces, upon inward product with any given whole real vector, four kinds of vectors: a) the same twodimensional real Mersenne vector, b) an imaginary-real one, c) a real-imaginary vector and d) the purely imaginary Mersenne vector. One can use the four vector complex characteristics summed upon a set in diverse ways, providing as a result vectors, which might be completely real, mixed real and complex, mixed imaginary and real, mixed imaginary and complex, completely complex and completely imaginary.

For instance, an $\mathrm{N}$-Dimensional version of a complex number can be constructed in the following way, using two whole real vectors

$$
\forall\{|\mathbf{a}\rangle,|\mathbf{b}\rangle\} \in V_{N}():|\mathbf{1}\rangle *|\mathbf{a}\rangle+|\mathbf{i}\rangle *|\mathbf{b}\rangle=|\mathbf{a}\rangle+i|\mathbf{b}\rangle=\left\{a_{I}+i b_{I} \mid I=1, N\right\},
$$


and the above expression can be written in this way above, because: $|\mathbf{i}\rangle=i|\mathbf{1}\rangle$.

The usual operations, like conjugation, described in complex algebra can be employed within this Complexity Hypercubes framework as in the scalar complex numbers case.

Vector spaces constructed using Complexity Hypercubes and whole vectors combine the character of the well-known Minkowski spaces, providing a generalization of them.

Of course, in the same way as there have been described vector classes following the number of null or equal elements entering the vector, the same organizing process could be associated to the vectors obtained from the vertices of a Complexity Hypercube.

Therefore, Complexity Hypercubes can be supposed to be in the definition of whole complex vectors.

\subsection{Geometrical considerations on the unity vectors}

Among the set of complex vectors constructed via Complexity Hypercubes one must consider the two vectors already described: $\{|\mathbf{1}\rangle,|\mathbf{i}\rangle \equiv i|\mathbf{1}\rangle\}$, as defining the unity vectors in real and pure imaginary subspaces.

Using Minkowski ideas, that is: not conjugating the imaginary unit when performing products, one can obtain the relative directions of both vectors. There are needed the scalar products:

$$
\langle\mathbf{1} \mid \mathbf{1}\rangle=N \wedge\langle\mathbf{i} \mid \mathbf{i}\rangle=-N \wedge\langle\mathbf{1} \mid \mathbf{i}\rangle=\langle\mathbf{i} \mid \mathbf{1}\rangle=i N
$$

they permit to compute the cosine of the subtended angle, like:

$$
\cos (\theta)=\frac{\langle\mathbf{1} \mid \mathbf{i}\rangle}{\sqrt{\langle\mathbf{1} \mid \mathbf{1}\rangle\langle\mathbf{i} \mid \mathbf{i}\rangle}}=\frac{i N}{\sqrt{N(-N)}}=\frac{i N}{i N}=1
$$

which constitutes an obvious result indicating that the unity vectors are collinear.

If conjugation is used as in the usual way scalar products in (pre-)Hilbert spaces are defined, then the scalar products become:

$$
\langle\mathbf{1} \mid \mathbf{1}\rangle=N \wedge\langle\mathbf{i} \mid \mathbf{i}\rangle=N \wedge\langle\mathbf{1} \mid \mathbf{i}\rangle=i N \wedge\langle\mathbf{i} \mid \mathbf{1}\rangle=\langle\mathbf{1} \mid \mathbf{i}\rangle^{*}=-i N
$$

providing a cosine like:

$$
\cos (\theta)=\frac{|\langle\mathbf{1} \mid \mathbf{i}\rangle|}{\sqrt{\langle\mathbf{1} \mid \mathbf{1}\rangle\langle\mathbf{i} \mid \mathbf{i}\rangle}}=\frac{N}{\sqrt{N N}}=1,
$$

a similar result as in the Minkowski case but considering that the module of the numerator has been employed to deal with real cosine values, as the result of the scalar products manipulation.

Squared Euclidian distance might be useful to complete the geometrical background of the meaning of both Mersenne real and imaginary vectors.

Owing to the definition of Euclidian distances as positive definite scalars, one needs to employ the usual scalar product conjugation to compute the distance in this case, producing:

$$
D^{2}=\langle\mathbf{1} \mid \mathbf{1}\rangle+\langle\mathbf{i} \mid \mathbf{i}\rangle-(\langle\mathbf{1} \mid \mathbf{i}\rangle+\langle\mathbf{i} \mid \mathbf{1}\rangle)=2 N-(i N+(-i) N)=2 N .
$$

Corresponding to a result reinforcing the collinearity of both unity vectors.

\section{Complete sum of a vector}

Suppose now an $N$-Dimensional vector space: $V_{N}(S)$, defined over some numerical set $S$. Then, the vectors of the space can be associated to the definitions:

$$
|\mathbf{a}\rangle \in V_{N}(S) \rightarrow|\mathbf{a}\rangle=\left\{a_{I} \mid I=1, N\right\} \rightarrow \forall I=1, N: a_{I} \in S
$$

The complete sum of a vector defined within $V_{N}(S)$ might be defined using the symbol \langle\rangle applied over a given vector or to a complicated set of vector operations yielding a vector. That is, the complete sum is constructed in such a way that:

$$
\left.\langle\mid \mathbf{a}\rangle\rangle=\sum_{I=1}^{N} a_{I} \rightarrow\langle\mid \mathbf{a}\rangle\right\rangle \in S
$$

The complete sum of a vector could be associated to some linear operator kind, as it is easy to prove that:

$$
\left.\left.\forall\left\{\left|\mathbf{a}_{J}\right\rangle\right\} \subset V_{N} \wedge\left\{\alpha_{J}\right\} \subset S:|\mathbf{s}\rangle=\sum_{J} \alpha_{J}\left|\mathbf{a}_{J}\right\rangle \Rightarrow\langle\mid \mathbf{s}\rangle\right\rangle=\sum_{J} \alpha_{J}\left\langle\mid \mathbf{a}_{J}\right\rangle\right\rangle .
$$

In a vector semispace, the resulting vector permutation Polyhedron, associated to any perfect vector, possess the obvious property of having the complete sum of every permutated whole vector equal to the complete sum of the perfect vector seed.

The complete sum of the vertex elements of any hollow class $\mathbf{C}_{\left[N_{1} ; N_{0}\right]}$ of a Boolean Hypercube $\mathbf{H}_{N}$ corresponds to $N_{1}$, that is the number of digits $\{1\}$ contained in every vertex of the class. 


\section{Generalized scalar products and norms}

Using the inward product of an indefinite number of vectors and applying the complete sum to the resultant vector, the scalar result of these two operations can be considered as a generalized scalar product.

Suppose a given Polyhedron defined over a vector space, defined in turn over some number set: $P=\left\{\left|\mathbf{a}_{K}\right\rangle \mid K=1, M\right\} \subset V_{N}(S)$, the $M$-th order scalar product corresponds to an element of the reference set $\mathrm{S}$, obtained by the complete sum of the inward product of the vectors of the Polyhedron. It might be defined accordingly as:

$$
\left.\langle P\rangle=\left\langle\begin{array}{c}
M \\
* \\
K=1
\end{array} \mid \mathbf{a}_{K}\right\rangle\right\rangle=\sum_{I=1}^{N}\left(\prod_{K=1}^{M} a_{I K}\right) \in S .
$$

Such a definition permits to construct the p-th norm of a vector: $|\mathbf{a}\rangle \in V_{N}(S)$, using the algorithm:

$$
\left.\left\langle\mid \mathbf{a}^{[p]}\right\rangle\right\rangle=\sum_{I=1}^{N}\left(a_{I}^{p}\right) ;
$$

that is, as the complete sum of the p-th inward power of a given vector.

\section{Inward power polyhedra generation}

Suppose a $M$-vertex Polyhedron: $P=\left\{\left|\mathbf{a}_{I}\right\rangle \mid I=1, M\right\}$, constructed in a $N$-Dimensional Vector Space $V_{N}(\mathbb{R})$, defined over the real field. One can write the Polyhedron as a row vector whose elements are the vertices of the Polyhedron and this circumstance might be expressed as:

$$
\left\langle P\left|=\left\langle\mid \mathbf{a}_{1}\right\rangle,\right| \mathbf{a}_{2}\right\rangle, \ldots\left|\mathbf{a}_{M}\right\rangle\left|=\left(\left|\mathbf{a}_{1}\right\rangle,\left|\mathbf{a}_{2}\right\rangle, \ldots\left|\mathbf{a}_{M}\right\rangle\right) .\right.
$$

The centroid of the Polyhedron might be simply defined as:

$$
|\mathbf{c}\rangle=M^{-1} \sum_{I}\left|\mathbf{a}_{I}\right\rangle
$$

With this information, a new translated Polyhedron: $T=\left\{\left|\mathbf{t}_{I}\right\rangle \mid I=1, M\right\}$ can be defined as:

$$
\forall I=1, M:\left|\mathbf{t}_{I}\right\rangle=\left|\mathbf{a}_{I}\right\rangle-|\mathbf{c}\rangle ;
$$

so, the centroid of the translated Polyhedron becomes null:

$$
|\mathbf{0}\rangle=M^{-1} \sum_{I}\left|\mathbf{t}_{I}\right\rangle
$$

Inward powers of the original polyhedron vertices can be also easily defined and written as:

$$
\left\langle P^{[p]}\left|=\left\langle\mid \mathbf{a}_{1}\right\rangle^{[p]},\right| \mathbf{a}_{2}\right\rangle^{[p]}, \ldots\left|\mathbf{a}_{M}\right\rangle^{[p]} \mid,
$$

with the implied inward powers constructed with the already defined algorithm:

$$
\forall I=1, M:\left|\mathbf{a}_{I}\right\rangle^{[p]}=\underset{k=1}{*}\left|\mathbf{a}_{I}\right\rangle=\left|\mathbf{a}_{I}\right\rangle *\left|\mathbf{a}_{I}\right\rangle * \ldots\left|\mathbf{a}_{I}\right\rangle=\left\{a_{J I}^{p} \mid J=1, N\right\} .
$$

Thus, the centroid of a general inward power Polyhedron might be written as:

$$
\left|\mathbf{c}^{(p)}\right\rangle=M^{-1} \sum_{I}\left|\mathbf{a}_{I}\right\rangle^{[p]}
$$

and the translated Polyhedron vertices might be described as:

$$
\forall I=1, M:\left|\mathbf{t}_{I}^{(p)}\right\rangle=\left|\mathbf{a}_{I}\right\rangle^{[p]}-\left|\mathbf{c}^{(p)}\right\rangle,
$$

providing the vertices of the translated Polyhedron:

$$
\left\langle T^{[p]}\left|=\left\langle\mid \mathbf{t}_{1}^{(p)}\right\rangle,\right| \mathbf{t}_{2}^{(p)}\right\rangle, \ldots\left|\mathbf{t}_{M}^{(p)}\right\rangle \mid
$$

Thus, in the same way as in the original Polyhedron, within the inward power translated Polyhedron it will also hold the property, consisting into that the centroids of any translated inward power Polyhedra are null:

$$
|\mathbf{0}\rangle=M^{-1} \sum_{I}\left|\mathbf{t}_{I}^{(p)}\right\rangle
$$

Therefore, knowing some inward power Polyhedra sequence:

$$
p=1,2, \ldots:\left\langle P^{[p]}\right|,
$$

there is a trivial matter to compute the corresponding centroid sequence:

$$
p=1,2, \ldots:\left|\mathbf{c}^{(p)}\right\rangle,
$$

which can be used to build up a set of translated Polyhedra:

$$
p=1,2, \ldots:\left\langle T^{[p]}\right|,
$$

whose centroids could be homogenously associated to the null vector $|\mathbf{0}\rangle$.

In this way, every member of the translated Polyhedra, contained into the inward power sequence, possess a common centroid coincident with the null vector. 


\subsection{Comparing vertex differences in polyhedra}

Differences between Polyhedron vertices might be simply obtained with usual tools.

For example, to represent with a vector the differences between first and second order translated Polyhedra can be done in the following way. Suppose known a translated vector: $|\mathbf{t}\rangle=|\mathbf{a}\rangle-|\mathbf{c}\rangle$ and the corresponding translated second inward power of it: $\left|\mathbf{t}^{(2)}\right\rangle=|\mathbf{a}\rangle^{[2]}-\left|\mathbf{c}^{(2)}\right\rangle$. It has been dropped the vertex subindex in order the symbol can be thought as representative of any Polyhedron vertex.

Scalar products are basic to obtain by further manipulation the possible differences. Now, considering that:|a $\rangle^{[2]}=|\mathbf{a}\rangle *|\mathbf{a}\rangle$ and that the scalar product can be easily expressed as the complete sum of an inward product of two vectors:

$$
\langle\mathbf{t} \mid \mathbf{a}\rangle=\langle\mid \mathbf{t}\rangle *|\mathbf{a}\rangle\rangle=\sum_{J} t_{J} a_{J}
$$

so, it can be written:

$$
\begin{aligned}
\left\langle\mathbf{t} \mid \mathbf{t}^{(2)}\right\rangle & \left.=\langle\mid \mathbf{a}\rangle *(|\mathbf{a}\rangle *|\mathbf{a}\rangle)\rangle-\left(\left\langle\mathbf{a} \mid \mathbf{c}^{(2)}\right\rangle+\langle\mid \mathbf{c}\rangle *(|\mathbf{a}\rangle *|\mathbf{a}\rangle)\right\rangle\right)+\left\langle\mathbf{c} \mid \mathbf{c}^{(2)}\right\rangle \\
& \left.\left.\left.\left.=\langle\mid \mathbf{a}\rangle^{[3]}\right\rangle-M^{-1} \sum_{I}\langle\mid \mathbf{a}\rangle *\left(\left|\mathbf{a}_{I}\right\rangle *\left|\mathbf{a}_{I}\right\rangle\right)\right\rangle-M^{-1} \sum_{I}\left\langle\mid \mathbf{a}_{I}\right\rangle *(|\mathbf{a}\rangle *|\mathbf{a}\rangle)\right\rangle+M^{-2} \sum_{I} \sum_{J}\left\langle\mid \mathbf{a}_{I}\right\rangle *\left(\left|\mathbf{a}_{J}\right\rangle *\left|\mathbf{a}_{J}\right\rangle\right)\right\rangle \\
& \left.\left.\left.\left.=\langle\mid \mathbf{a}\rangle^{[3]}\right\rangle-M^{-1} \sum_{I}\langle\mid \mathbf{a}\rangle *\left|\mathbf{a}_{I}\right\rangle^{[2]}\right\rangle-M^{-1} \sum_{I}\left\langle\mid \mathbf{a}_{I}\right\rangle *|\mathbf{a}\rangle^{[2]}\right\rangle+M^{-2} \sum_{I} \sum_{J}\left\langle\mid \mathbf{a}_{I}\right\rangle *\left|\mathbf{a}_{J}\right\rangle^{[2]}\right\rangle
\end{aligned}
$$

This construction above might be considered most interesting, because the centroids of the sequence of inward powers $\left\langle P^{[p]}\right|$ are alternatively attached to a sequence of centroids $\left|\mathbf{c}^{(p)}\right\rangle$, which is far from being homogeneous.

Every term in the above equation corresponds to a scalar product of three vectors, which in general can be written as:

$$
\left.\forall|\mathbf{a}\rangle,|\mathbf{b}\rangle,|\mathbf{c}\rangle \in V_{N}:\langle\mid \mathbf{a}\rangle *|\mathbf{b}\rangle *|\mathbf{c}\rangle\right\rangle=\sum_{I=1}^{N} a_{I} b_{I} c_{I} .
$$

Therefore, using the previous definitions of the preceding section, for instance:

$$
\left.\langle\mid \mathbf{a}\rangle^{[3]}\right\rangle=\sum_{I=1}^{N} a_{I}^{3}
$$

might represent a third order Minkowski pseudonorm of the vector $|\mathbf{a}\rangle$.

\section{Statistical-like vectors and power series of a polyhedron}

The centroid inhomogeneity of powers of a Polyhedron might be instead associated to the definition of a sequence of statistical-like vectors, which can be seen, in turn, as an $\mathrm{N}$-Dimensional generalization of the well-known statistical moments, which could be connected to any set of scalar values of an aleatory variable.

The first element of this Polynomial inward power sequence corresponds to the null vector, which can be associated on the other hand to the mean value vector of the translated original Polyhedron vertices. While the second inward power term is easily associated to a vector, which corresponds to the generalization of the variance. This variance-like vector might be written as the centroid difference and further simplified:

$$
\left|\mathbf{m}^{(2)}\right\rangle=M^{-1} \sum_{I}\left(\left|\mathbf{a}_{I}\right\rangle-|\mathbf{c}\rangle\right)^{[2]}=\left|\mathbf{c}^{(2)}\right\rangle-|\mathbf{c}\rangle^{[2]} .
$$

Higher order statistical-like moments can be written in the same way:

$$
p=1,2, \ldots:\left|\mathbf{m}^{(p)}\right\rangle=M^{-1} \sum_{I}\left(\left|\mathbf{a}_{I}\right\rangle-|\mathbf{c}\rangle\right)^{[p]}=M^{-1} \sum_{k=0}^{p}(-1)^{k}\left(\begin{array}{c}
p \\
k
\end{array}\right) \sum_{I}\left(\left|\mathbf{a}_{I}\right\rangle^{[p-k]} *|\mathbf{c}\rangle^{[k]}\right),
$$

which can be simplified using a bit more involved expression:

$$
p=1,2, \ldots:\left|\mathbf{m}^{(p)}\right\rangle=\left|\mathbf{c}^{(p)}\right\rangle+\delta(p>2) M^{-1} \sum_{k=1}^{p-2}(-1)^{k}\left(\begin{array}{c}
p \\
k
\end{array}\right) \sum_{I}\left(\left|\mathbf{a}_{I}\right\rangle^{[p-k]} *|\mathbf{c}\rangle^{[k]}\right)+(-1)^{p-1}(p-1)|\mathbf{c}\rangle^{[p]} .
$$

Note that, whenever the inward powers defining the statistical-like vector moments are obtained with the inward absolute power of the differences involved:

$$
\left.p=1,2, \ldots:\left|\mathbf{d}^{(p)}\right\rangle=M^{-1} \sum_{I}|| \mathbf{a}_{I}\right\rangle-\left.|\mathbf{c}\rangle\right|^{[p]},
$$

using the inward function concept of a vector as follows:

$$
\forall|\mathbf{a}\rangle=\left\{a_{I} \mid I=1, N\right\} \rightarrow f[|\mathbf{a}\rangle]=\left\{f\left(a_{I}\right) \mid I=1, N\right\},
$$

then, the sequence of vectors: $\left\{\left|\mathbf{d}^{(p)}\right\rangle \mid p=1,2, \ldots\right\}$ belongs to a vector semispace. The complete sum of each vector in the above sequence of statistical vector moments, describes a positive definite scalar or a $p$-th order Minkowski norm:

$$
\left.p=1,2, \ldots:\left\langle\mid \mathbf{d}^{(p)}\right\rangle\right\rangle=m_{p}
$$


The scalar set generated in this way also can be supposedly imagined that corresponds to the collective distances of increasing order, involving all the original Polyhedron vertices. One might note that the even inward powers of both sequences are equivalent in both cases, that is:

$$
p=1,2, \ldots:\left|\mathbf{d}^{(p)}\right\rangle=\left|\mathbf{m}^{(p)}\right\rangle .
$$

Such kind of vector momenta sequences cannot be defined within the translated inward power Polyhedra. They shall be considered as another inward power sequence associated just to the first order translated Polyhedron: $T=\left\{\left|\mathbf{t}_{I}\right\rangle \mid I=1, M\right\}$.

$$
p=1,2, \ldots:\left\langle S^{[p]}\left|=\left\langle\mid \mathbf{t}_{1}\right\rangle^{[p]},\right| \mathbf{t}_{2}\right\rangle^{[p]}, \ldots\left|\mathbf{t}_{M}\right\rangle^{[p]} \mid,
$$

corresponding to an inhomogeneous centroid sequence of inward power Polyhedra. Such centroids coincide with the set of statistical-like momenta.

Computed in any way, the sequence of complete sums of the statistical-like vectors of a given Polyhedron generates a vector which might be considered as a vector defining the Polyhedron in a condensed form. In this way two or more polyhedra can be compared.

\subsection{Polyhedron power series}

The homogeneous centroid inward power Polyhedra, might be employed to describe a Polyhedron series which can be expressed in the simple form:

$$
\left\langle Z\left|=\sum_{p} \theta_{p}\left\langle T^{[p]}|\rightarrow \forall I=1, M:| \mathbf{z}_{I}\right\rangle=\sum_{p} \theta_{p}\right| \mathbf{t}_{I}\right\rangle^{[p]} .
$$

The vertices of the Polyhedron described by the above series might be used as operators acting on the original Polyhedron vertices in such a manner that one can write the scalar products between the series Polyhedron vertices and the original Polyhedron ones:

$$
\forall I=1, M:\left\langle\mathbf{z}_{I} \mid \mathbf{a}_{I}\right\rangle=\pi_{I} \equiv \sum_{p} \theta_{p}\left({ }^{[p]}\left\langle\mathbf{t}_{I} \mid \mathbf{a}_{I}\right\rangle\right) .
$$

The scalar products appearing in the right-hand side of the above expression can be taken, as it has been already commented, as generalized scalar products. To make clearer this last statement, one can write the simple scalar product by means of the complete sum of the inward product of two vectors, as discussed before. Then, according to the already described inward powers, a generalized scalar product involving several vectors can be defined just in the following way:

$$
\left.\left.[p]\langle\mathbf{t} \mid \mathbf{a}\rangle=\langle\mid \mathbf{t}\rangle^{[p]} *|\mathbf{a}\rangle\right\rangle=\langle(\underset{p}{*}|\mathbf{t}\rangle) * \mid \mathbf{a}\rangle\right\rangle=\sum_{J} t_{J}^{p} a_{J}
$$

\subsection{Expression of an expectation value}

Therefore, the equality:

$$
\forall I=1, M: \pi_{I} \equiv \sum_{p} \theta_{p}\left({ }^{[p]}\left\langle\mathbf{t}_{I} \mid \mathbf{a}_{I}\right\rangle\right)=\sum_{p} \theta_{p} Z_{I p}
$$

might be transformed easily into a linear matrix equation:

$$
|\pi\rangle=\mathbf{Z}|\theta\rangle
$$

with obvious definitions:

$$
|\pi\rangle=\left\{\pi_{I}\right\} ;|\theta\rangle=\left\{\theta_{p}\right\} ; \mathbf{Z}=\left\{Z_{I p}\right\} .
$$

If the $|\pi\rangle$ vector components are well-defined and known, then the components of the coefficient vector $|\theta\rangle$ might be computed if there are some sound solutions of the associated equation (9.1).

\subsection{Exponential approximation}

Another possibility might be found using directly an inward exponential function with the translated original Polynomial vertices as variable:

$$
\forall I=1, M:\left|\mathbf{z}_{I}\right\rangle=\exp \left(-\alpha\left|\mathbf{t}_{I}\right\rangle\right)=\sum_{p=0}^{\infty} \frac{(-\alpha)^{p}}{p !}\left|\mathbf{t}_{I}\right\rangle^{[p]}=|\mathbf{1}\rangle-\alpha\left|\mathbf{t}_{I}\right\rangle+\frac{\alpha^{2}}{2}\left|\mathbf{t}_{I}\right\rangle^{[2]}+O(3)
$$

the exponential parameter value might be obtained as follows. Using the exponential vectors defined in equation (9.2). operator-like, then:

$$
\forall I=1, M:\left\langle\mathbf{z}_{I} \mid \mathbf{a}_{I}\right\rangle=\pi_{I} \approx\left\langle\mathbf{1} \mid \mathbf{a}_{I}\right\rangle-\alpha\left\langle\mathbf{t}_{I} \mid \mathbf{a}_{I}\right\rangle+\frac{\alpha^{2}}{2}\left({ }^{[2]}\left\langle\mathbf{t}_{I} \mid \mathbf{a}_{I}\right\rangle\right)+O(3)
$$

therefore, if it exists, the exponential parameter $\alpha$ might be computed by solving:

$$
\forall I=1, M:\left\langle\mathbf{1} \mid \mathbf{a}_{I}\right\rangle-\pi_{I}-\alpha\left\langle\mathbf{t}_{I} \mid \mathbf{a}_{I}\right\rangle+\frac{\alpha^{2}}{2}\left({ }^{[2]}\left\langle\mathbf{t}_{I} \mid \mathbf{a}_{I}\right\rangle\right)=0
$$

which can be written in general, dropping the vertex indices, like:

$$
\tau_{0}-\pi-\tau_{1} \alpha+\frac{\tau_{2}}{2} \alpha^{2}=0 \rightarrow \alpha=\frac{1}{\tau_{2}}\left(\tau_{1} \pm \sqrt{\left(\tau_{1}\right)^{2}+2 \tau_{2}\left(\pi-\tau_{0}\right)}\right)
$$

Of course, taking more terms in equation (9.3) the problem corresponds to obtain the roots of a higher order polynomial in $\alpha$. It must be noted too that the successive inward powers of the translated vertices correspond to the original Polynomial non-averaged moments. 


\section{Linear independence of inward powers of a whole vector}

Another question might be proposed as follows. Are two inward powers of the same vector linearly independent? For instance: $|\mathbf{a}\rangle$ and $|\mathbf{a}\rangle^{[2]}$ are in general linearly independent? To test this issue it is easy, because if they are linearly independent the equation:

$$
\alpha|\mathbf{a}\rangle+\beta|\mathbf{a}\rangle^{[2]}=|\mathbf{0}\rangle
$$

implies that $\alpha=\beta=0$. Therefore, to test the truth of this double equality, the previous equation can be rewritten as:

$$
\alpha|\mathbf{a}\rangle+\beta(|\mathbf{a}\rangle *|\mathbf{a}\rangle)=|\mathbf{0}\rangle
$$

and furthermore, the equality can be rearranged owing to the nature of the inward product and the existence of the unity vector: $|\mathbf{1}\rangle=$ $\left\{1_{I}=1 \mid I=1, N\right\}$, which acts as neutral element of the inward product, besides taking: $|\mathbf{a}\rangle \neq|\mathbf{0}\rangle$, or assuming it is a perfect or whole vector, then one can write:

$$
|\mathbf{a}\rangle *(\alpha|\mathbf{1}\rangle+\beta|\mathbf{a}\rangle)=|\mathbf{0}\rangle \rightarrow \alpha|\mathbf{1}\rangle+\beta|\mathbf{a}\rangle=|\mathbf{0}\rangle \rightarrow \alpha|\mathbf{1}\rangle=-\beta|\mathbf{a}\rangle,
$$

thus, whenever $|\mathbf{a}\rangle \neq \lambda|\mathbf{1}\rangle$, one arrives to the fact that necessarily: $\alpha=\beta=0$, and then this implies the linear independence of the two inward powers.

This result can be easily generalized writing:

$$
p<q: \alpha|\mathbf{a}\rangle^{[p]}+\beta|\mathbf{a}\rangle^{[q]}=|\mathbf{0}\rangle \rightarrow|\mathbf{a}\rangle^{[p]} *\left(\alpha|\mathbf{1}\rangle+\beta|\mathbf{a}\rangle^{[q-p]}\right)=|\mathbf{0}\rangle
$$

Thus, a similar argument as before can be employed, as if:

$$
|\mathbf{a}\rangle \neq \lambda|\mathbf{1}\rangle \rightarrow|\mathbf{a}\rangle^{[q-p]} \neq \lambda|\mathbf{1}\rangle
$$

If any pair of powers of a whole vector are linearly independent, any Polyhedron made of powers of a unique vector can furnish linearly independent generators, basis sets of any given vector space.

However, there appears just here a paradox, because if the successive inward powers, which can be written as: $\{|\mathbf{a}\rangle[p] \mid p=1,2, \ldots n, \ldots\}$, of a non-unity vector are linearly independent, then in case the vectors belong to some vector space $V_{N}$, just a limited number $N$ of them can be taken together as a linearly independent set generating the space, a basis set, and it can be written, say: $\left\{|\mathbf{a}\rangle^{[p]} \mid p=1,2, \ldots N\right\}$ as a linearly independent set. Even one can think about using a first term in the series: $|\mathbf{a}\rangle^{[0]}=|\mathbf{1}\rangle$ in case of need or fancy, but then of course, the linearly independent set will be written as:

$$
\left\{|\mathbf{a}\rangle^{[p]} \mid p=0,2, \ldots, N-1\right\}
$$

This situation might be easily expressed in general, for instance, using:

$$
\forall q>N, \exists\left\{\alpha_{p}^{(q)} \mid p=1, N\right\}: \sum_{p=1}^{N} \alpha_{p}^{(q)}|\mathbf{a}\rangle^{[p]}=|\mathbf{a}\rangle^{[q]}
$$

If the seed vector of any Power Polyhedron, like the one previously defined, is a whole or perfect vector, then inverse powers are also allowed to construct such classes of Polyhedra.

When dealing with vector spaces $V_{N}$, of dimension $N$, then just a limited number $N$ of linearly independent vectors form a basis set. This means that any power of a given vector can be constructed with the $N$ chosen power vectors of the basis set.

This means that, for instance, in 2-dimensional spaces the whole vectors: $|\mathbf{a}\rangle$ and $|\mathbf{a}\rangle^{[2]}$ can generate any inward power of $|\mathbf{a}\rangle$, including the Mersenne vertex. This can be set up like the set of equivalent equalities:

$$
|\mathbf{1}\rangle=\alpha|\mathbf{a}\rangle+\beta|\mathbf{a}\rangle^{[2]} \rightarrow|\mathbf{1}\rangle=|\mathbf{a}\rangle *(\alpha|\mathbf{1}\rangle+\beta|\mathbf{a}\rangle) \rightarrow|\mathbf{a}\rangle^{[-1]}=\alpha|\mathbf{1}\rangle+\beta|\mathbf{a}\rangle
$$

meaning that:

$$
\left.\left.\begin{array}{l}
a_{1}^{-1}=\alpha+\beta a_{1} \\
a_{2}^{-1}=\alpha+\beta a_{2}
\end{array}\right\} \Rightarrow \quad \begin{array}{c}
\frac{a_{1}^{-1}-a_{2}^{-1}}{a_{1}-a_{2}}=\beta \\
\frac{1}{2}\left[\left(a_{1}^{-1}+a_{2}^{-1}\right)-\beta\left(a_{1}+a_{2}\right)\right]=\alpha
\end{array}\right\}
$$

Moreover, such a composition might be done with any pair of powers of the vector $|\mathbf{a}\rangle$. Of course, any vector of the 2-dimensional space might be generated with any pair of powers of the vector.

The following statements might be considered. Any perfect or whole vector $|\mathbf{a}\rangle$ of any $N$-dimensional space $V_{N}$ can be used to generate a basis set, based uniquely on the vector $|\mathbf{a}\rangle$. A basis set of $V_{N}$ can be constructed using $N$ arbitrary but different inward powers of the perfect or whole vector $|\mathbf{a}\rangle$. That is the same to say that, considering some vector space $V_{N}$, then any Polyhedron made by $N$ distinct inward powers of an arbitrary whole vector, corresponds to a basis set of $V_{N}$. 


\section{Conclusions}

Boolean Hypercubes might schematically contain the structure of any vector space. The concept of model or seed vertices of the Hypercube permit to construct vertex classes, which might have a similar reference in vector spaces. Perfect vectors can be considered the true elements of any vector space from where $N$ ! whole (that is: not bearing null elements) vectors can be deduced. While hollow (that is: bearing null elements) vectors might be considered vectors of spaces of lesser dimensions. Signature Hypercubes can be employed via inward products as possible sign generators, which can be attached in turn to any whole vector. Such construct can be employed in many instances, like the construction of Minkowski spaces. The Polyhedron obtained by the inward powers of a whole vector, can be used to construct a basis set of the attached vector space. Inward products involving vectors and the complete sum of a vector might be employed in vector spaces to describe generalized scalar products, hence also vector norms. Statistical-like vectors of Polyhedra contained in a vector space can be easily defined. They can lead, via their complete sum, to a set of scalars, which ordered as a vector of arbitrary dimensions can resume in a condensed manner the attached Polyhedron. From these considerations, a new look into vector spaces emerges. Application of the developed theoretical frame might have powerful applications into quantum QSPR and beyond, that is: in the characterization and comparison of molecular sets represented, as it is customary, in the form of finite dimensional vectors.

\section{References}

[1] S. K. Berberian, Introduction to Hilbert Space, Oxford University Press, 1961.

[2] F. Ayres Jr., Matrices, Schaum Pub. Co. (Mc Graw Hill), 1962.

[3] G. E. Shilov, Linear Algebra, Dover Publications Inc. (New York), 1971

[4] H. Schneider, G. Ph. Barker, Matrices and Linear Algebra, Dover Publications Inc. (New York), 1989.

[5] P. O. Löwdin, Linear Algebra for Quantum Theory, J. Wiley \& Sons, 1998.

[6] G. A. Korn, Th. M. Korn, Mathematical Handbook for Scientists and Engineers, Dover Publications Inc. (New York), 2000.

[7] L. Hogben (Editor), Handbook of Linear Algebra, Chapman \& Hall/CRC Taylor \& Francis Group (Boca Raton), 2007.

[8] T. Gowers (Editor), The Princeton Companion to Mathematics, Princeton Univ. Press (Princeton), 2008.

[9] R. Carbó-Dorca, On the statistical interpretation of Density Functions: ASA, Convex Sets, discrete Quantum Chemical molecular representations, Diagonal Vector Spaces and related problems, J. Math. Chem. 23 (1998), 365-375.

[10] R. Carbó-Dorca, Shell partition and metric semispaces: Minkowski norms, root scalar products, distances and cosines of arbitrary order, J. Math. Chem. 32 (2002), 201-223.

[11] P. Bultinck and R. Carbó-Dorca, A mathematical discussion on density and shape functions, vector semispaces and related questions, J. Math. Chem. 36 (2004), 191-200.

[12] R. Carbó-Dorca and S. Van Damme, Riemann spaces, molecular density function semispaces, quantum similarity measures and quantum quantitative structure-properties relationships (QQSPR), An Homage to Dr. J. J. Bonet, Afinidad 64 (2007), 147-153.

[13] R. Carbó-Dorca, Molecular Quantum Similarity Measures in Minkowski Metric Vector Semispaces, J. Math. Chem. 40 (2008), 628-636.

[14] R. Carbó-Dorca and E. Besalú, Shells, point cloud huts, generalized scalar products, cosines and similarity tensor representations in vector Semispaces, J. Math. Chem. 50 (2012), 210-219.

[15] R. Carbó-Dorca, Natural Vector Spaces, (Inward Power and Minkowski Norm of a Natural Vector, Natural Boolean Hypercubes) and Fermat's Last Theorem, J. Math. Chem. 55 (2017), 914-940

[16] R. Carbó-Dorca, C. Muñoz-Caro, A. Niño and S. Reyes, Refinement of a Generalized Fermat's Last Theorem Conjecture in Natural Vector Spaces, J. Math. Chem. 55 (2017) 1869-1877.

[17] R. Carbó-Dorca, Multimolecular Polyhedra and QSPR, J. Math. Chem. 52 (2014), 1848-1856.

[18] R. Carbó-Dorca, Quantum Polyhedra, Definitions, Statistics and the Construction of a Collective Quantum Similarity Index, J. Math. Chem. Vol. 53, pp $171-182,2015$

[19] R. Carbó-Dorca and D. Barragán, Communications on Quantum Similarity (4): Collective distances computed by means of Similarity Matrices, as generators of intrinsic ordering among Quantum Multimolecular Polyhedra, WIREs Comput. Mol. Sci. 5, (2015) $380-404$.

[20] R. Carbó-Dorca, An Isometric Representation Problem Related with Quantum Multimolecular Polyhedra and Similarity, J. Math. Chem. 53 (2015), $1750-1758$

[21] R. Carbó-Dorca, An Isometric Representation Problem in Quantum Multimolecular Polyhedra and Similarity: (2) Synisometry, J. Math. Chem. 53 (2015), 1867-1884.

[22] R. Carbó-Dorca, Ouantum Molecular Polyhedra in LCAO-MO Theory, Mol. Phys. 114 (2016), 1236-1249.

[23] R. Carbó-Dorca and S. González, Notes in QSPR (4): Quantum Multimolecular Polyhedra, Collective Vectors, Quantum Similarity and Quantum QSPR fundamental equation, Management Studies 4 (2016), 33-47.

[24] R. Carbó-Dorca, A Study on the Centroid Vector of a Polyhedron, J. Math. Chem 54 (2016), 51-71.

[25] R. Carbó-Dorca, Time Vectors and Particle Swarms Defined as Polyhedra in Spherically Enfolded Spaces, J. Math. Chem. 54 (2016), 1751-1757.

[26] R. Carbó-Dorca, A Theorem on the Gram matrix of a Polyhedron, J. Math. Chem. 55 (2017), 79-97.

[27] R. Carbó-Dorca, Fuzzy sets and Boolean Tagged sets, J. Math. Chem. 22 (1997), 143-147.

[28] R. Carbó-Dorca; Fuzzy sets and Boolean Tagged sets, vector semispaces and convex sets, QSM and ASA density functions, diagonal vector spaces and quantum Chemistry, Adv. Molec. Simil., JAI Press, 2 (1998), 43-72.

[29] R. Carbó-Dorca, About Erdös Discrepancy Conjecture, J. Math. Chem. 54 (2016), 657-660.

[30] R. Carbó-Dorca, N-dimensional Boolean Hypercubes and the Goldbach Conjecture, J. Math. Chem. 54 (2016), 1213-1220.

[31] R. Carbó-Dorca, A study on Goldbach Conjecture, J. Math. Chem. 54 (2016), 1798-1809.

[32] R. Carbó-Dorca, Boolean Hypercubes as Time Representation Holders, J. Math. Chem. 56 (2018), 1349-1352.

[33] R. Carbó-Dorca, About some questions relative to the arbitrariness of signs: Their possible consequences in matrix signatures definition and quantum chemical applications, J. Math. Chem. 33 (2003), 227-244.

[34] R. Carbó-Dorca, Inward Matrix Products: Extensions and Applications to Quantum Mechanical Foundations of QSAR, J. Mol. Struct. (Teochem) 537 (2001), 41-54.

[35] R. Carbó-Dorca, Inward Matrix product algebra and calculus as tools to construct space-time frames of arbitrary dimensions, J. Math. Chem. 30 (2001), 227-245

[36] R. Carbó-Dorca, Applications of Inward Matrix Products and Matrix Wave Functions to Hückel MO Theory, Slater Extended Wave Functions, Spin Extended Functions and Hartree Method, Int. J. Quantum Chem. 91 (2003), 607-617. 